\title{
Rethinking organizational change in the COVID-19 era
}

\author{
Amanda Choflet ${ }^{1}$, Thomas Packard ${ }^{2}$, Keren Stashower ${ }^{3}$ \\ ${ }^{1}$ School of Nursing, San Diego State University, San Diego, CA, USA; ${ }^{2}$ School of Social Work, San Diego State University, San Diego, CA, USA; \\ ${ }^{3}$ Kinnectics, LLC, San Diego, CA, USA \\ Contributions: (I) Conception and design: All authors; (II) Administrative support: None; (III) Provision of study materials or patients: None; (IV) \\ Collection and assembly of data: None; (V) Data analysis and interpretation: All authors; (VI) Manuscript writing: All authors; (VII) Final approval of \\ manuscript: All authors. \\ Correspondence to: Thomas Packard. 261 Hygeia Ct. Encinitas, CA 92024, USA. Email: tpackard@sdsu.edu.
}

\begin{abstract}
Planned organizational change has a long history in formal organizations of all types. A model of planned organizational change from the existing literature can be used to identify a set of principles which can be offered for use during the current COVID-19 crisis, but this traditional model may not be fully adequate during this especially challenging period. A relatively new model from the field of complexity theory for organizations, Complex Adaptive Systems (CAS), offers promise for addressing the unique crisis conditions facing hospitals in the COVID-19 era. These conditions require constant assessment; ongoing high levels of communication; and iterative cycles of experimentation, reflection and learning, which can be better addressed in a CAS framework. Healthcare providers are experiencing periods of relative calm and interspersed crisis, which need to be proactively recognized and managed through sustained, "big picture" changes using timely combinations of traditional methods, complexity approaches, with the flexibility and ethical considerations of crisis management strategies built in. Successful crisis management requires leadership skills of those involved in decision-making at the local level, with a preference for the use of inclusive and adaptive leadership styles. Innovative strategies, teamwork, and crisis management models that have recently emerged may enable deeper systemic change in the long term. Any hospital or program in a healthcare system should be able to use this material to address a significant organizational change need.
\end{abstract}

Keywords: Organizational change; COVID-19; crisis management; Complex Adaptive Systems (CAS); healthcare

Received: 29 September 2020; Accepted: 18 February 2021; Published: 25 June 2021.

doi: 10.21037/jhmhp-21-11

View this article at: http://dx.doi.org/10.21037/jhmhp-21-11

\section{Introduction}

The COVID-19 crisis has created unprecedented demands for immediate and far-reaching organizational change in every healthcare delivery institution. Employees from executive to frontline staff are grappling with the pace, breadth, and depth of these demands. The field and practices of organizational change are being heavily challenged in terms of needs for change and pressures to make it happen more quickly than ever. The purpose of this paper is to consider how the demands of the COVID-19 era are affecting organizational change practice, including how traditional and evidence-based methods are or are not being used, and how the field needs to change in order to adapt to this new reality.

A brief review of the organizational change literature will be followed by a discussion of organizational change principles that will be used to provide a framework for assessing current practices in hospitals to deal with the COVID-19 crisis. Complex Adaptive Systems (CAS) will be offered as an augmentation to traditional change principles. Current issues and challenges will be discussed, followed by a model which blends the two approaches. A conclusion will offer suggestions on how to address this and future crises.

\section{Literature review}

Organizational change is broadly seen as any effort to, in 
a planned way, modify the operations or processes of an organization. Its implementation ranges from facilitation by highly experienced organization development consultants to managers giving orders for staff to do something in a new way.

There is a vast literature on organizational change, mainly in the general business or organizational behavior fields, ranging from books in the popular management press to scholarly articles. Key elements from the generic organizational change and change leadership literatures are summarized here, followed by current literature on organizational change in hospitals. Much of this literature focuses on theory development, studies with only a limited number of possible variables, or individual case studies, offering limited guidance to actual practice. These literatures include conceptual frameworks, theoretical considerations, studies of selected variables in the change process, qualitative and quantitative methods, and practice guidelines, but rarely in the same source. Practice guidelines are often presented without a conceptual framework, with evidence limited to the author's authority or experiences as a consultant, or are limited to findings from a single case study. Specific organizational change strategies, tactics, and methods are rarely examined in a detailed and systematic way.

Parry, cited in Hughes (1), offered this summary: 'organizational change' has become an interest for organizational consultants more so than for empirical researchers. There are many more books and articles on practitioner or conceptual scholarship than on theoretical or empirical scholarship. Much of the practitioner work is case study-based, and anecdotal and not rigorous in its conduct.

The organizational change literature in healthcare follows the field's general trends and lacks a comprehensive conceptual framework for studying the possible range of factors, variables, or change methods that can be used to identify specific success factors for organizational change. This gap in theoretical approaches to healthcare-specific organizational change has been noted as a key design flaw in healthcare organizational change efforts (2). Beginning in the 1980s, change focused mergers/restructuring with an emphasis on financial performance $(3,4)$. In most cases, the primary focus is simple: improve quality and outcomes while using fewer resources.

These "pay for performance," or "value-based incentives," have served as powerful motivators in healthcare organizations to move to new services or redesign existing programs with mixed outcomes regarding actual improvements in quality and cost $(5,6)$. Bundled payments, long considered an improvement over fee-for-service reimbursement models, have been shown to forcefully drive changes in care delivery which do not necessarily result in improvements in quality or reductions in the actual cost to the healthcare system (7). Some researchers have reported variable responses to the implementation of value-based incentives across the U.S., with some healthcare organizations serving the poor at increased risk for financial penalties incurred by uncompensated organizational change (8). Others have noted that quality improvements seen with participation in value-based reimbursement models do not necessarily incur cost savings (9).

The role of leadership within organizational change models in healthcare organizations continues to be discussed. Some studies regarding change leadership specifically have been reported recently (10). Øygarden et al. (11) referenced Yukl's model of change-oriented leadership behaviors as including monitoring and interpreting the environment, envisioning new possibilities for the organization, explaining the need for change, suggesting new and creative solutions and experimenting with new approaches for achieving objectives, taking a long-term perspective on problems and opportunities and negotiating for support from other actors on behalf of the department.

They concluded that "it is more reasonable to interpret our finding as an indication that organizational changes which in the current health policy climate are often motivated by cutting costs and increasing control and efficiency may indeed create more work system performance obstacles" (11), adding that "participation in decisionmaking is an aspect of such engagement and should be encouraged and safeguarded by hospital leadership in change processes as well as in day-to-day operations" (11). There is literature that examines organizational change in hospitals in terms of change tactics and methods suggesting that those at lower levels should be given as much autonomy and flexibility as possible to implement change at their levels $(12,13)$.

\section{A traditional planned change model}

As the literature review suggests, there does not seem to be a comprehensive conceptual framework for studying the possible range of factors, variables, or change methods that can be used to identify specific success factors regarding organizational change. There are evidence-based models of leadership that have particular relevance for organizational change, but these have not often been examined with 
specific attention to change leadership as distinct from day to day leadership.

A conceptual model of organizational change from the human services field (14) will be used here as a framework to consider how the existing literature is or is not being reflected in current COVID-19-related change efforts in hospitals. This model includes assessing the needs for change and internal factors of readiness and capacity for change, change goals, the scope of change, overall change strategy, and change tactics.

\section{Assessment}

Before a formal plan for change is introduced to staff, managers should conduct a comprehensive assessment of present conditions, including the specific environmental demands facing the organization and the current state of the organization in terms of existing capacities and readiness for change. Environmental demands based on the COVID-19 crisis are vivid, and do not need elaboration here. Internal assessments can consider the organization's readiness for change and existing capacities to implement change. The principles and bullet points in the next section can be used by the organization's change leaders as criteria to identify areas of capacity and readiness that may need to be addressed and improved before launching the change initiative.

\section{Readiness and capacity for change}

Readiness for change includes "the extent to which an individual or individuals are cognitively and emotionally inclined to accept, embrace, and adopt a particular plan to purposefully alter the status quo" (15).

Change leader readiness and capacity for change can be assessed with reference to characteristics including a tolerance for ambiguity, accepting not being able to control everything, understanding how feelings affect behavior, and being open to shared decision making (16). Tolerance for ambiguity is especially important during the COVID-19 crisis as change leaders are confronted with multiple interconnected uncertainties, conflicting demands from stakeholders, and resource inadequacies, Accepting not being able to control everything suggests that a participative management philosophy could be appropriate. Burke added that "effective change leaders need to have an aboveaverage level of energy and be capable of (I) working long hours when needed, (II) interacting with lots of people, and
(III) energizing others" (16).

In the midst of a crisis, all of those characteristics would ideally be present as preconditions for success. Individual leaders could assess themselves and work to add or improve their competencies while change is occurring. Inadequate change leadership skills can be corrected in the future through leader training and development.

Staff readiness at an individual or group level can include:

* Perceiving the need for change;

* Feelings of confidence regarding one's change skills;

* Feeling that the change will be personally beneficial (15).

The first item should be obvious to staff during the current crisis. The others may be affected by the way staff are led and communicated with.

Factors regarding organizational readiness for change include:

* Motivation for change, including perceived needs or pressures for improvement;

* Adequacy of resources, including equipment, staffing, and training;

- Staff attributes including their feelings of self efficacy and adaptability;

* Organizational climate, including staff cohesiveness and trust, and management openness to suggestions from staff in making changes (17).

Similar to staff readiness, the first factor may have to be taken as a given, with the others varying based upon how the organization has prepared for the current crisis. Adequate resources, such as personal protective equipment (PPE) and ventilators, were apparently almost universal readiness weaknesses in the early weeks of the COVID-19 crisis. There are likely to be major variations among hospitals regarding all other aspects of readiness. Ideally, these conditions would have been already established, through adequate provision of resources and participative leadership styles which would have helped create staff confidence, cohesiveness, and trust in management. To the extent that these factors are missing or weak, change leaders will need to address these in the midst of creating change. At the policy level, readiness in terms of resources such as supply reserves has been a major problem, and at least should be kept in mind to prepare for future crises.

Staff attributes and organizational climate may be affected by leadership behavior.

Change capacity can be defined as "a combination of managerial and organizational capabilities that allows an 
enterprise to adapt more quickly and effectively than its competition to changing situations" (18).

Individual staff capacity for change flows from the organizational readiness factors listed above. Factors here include:

* Staff seeing the change as fitting with the vision and mission of the organization;

* Staff commitment to the purpose, value, and necessity of the change;

- Staff willingness to go along with the change;

* Staff job satisfaction, commitment to the organization, and trust in management;

* The level of staff autonomy or feelings of empowerment;

* Staff tolerance of ambiguity, which is likely to increase in times of change.

Organizational capacity for change can include these factors:

* Trustworthy leaders. The ability of senior executives to earn the trust of the rest of the organization and to show organizational members the way to meet its collective goals;

* Trusting followers. The ability of the non-executive employees to constructively dissent with and/or willingly follow a new path advocated by its senior executives;

* Capable champions. The ability of an organization to attract, retain, and empower change leaders to evolve and emerge;

* Involved mid-management. The ability of middle managers to effectively link senior executives with the rest of the organization;

* Innovative culture. The ability of the organization to establish norms of innovation and encourage innovative activity;

- Accountable culture. The ability of the organization to carefully steward resources and successfully meet predetermined deadlines;

* Effective communication. The ability of the organization to communicate vertically, horizontally, and with customers;

* Systems thinking. The ability of the organization to focus on root causes and recognize the interdependencies within and outside the organizational boundaries (18).

Readiness and capacity could already have been established through earlier leadership behaviors. For elements which are lacking or inadequate, change leaders can help improve them before or during change implementation.

\section{Change goals}

Goals of organizational change are typically based on responses to external or internal pressures for improvement. Examples include implementing new policies such as the Affordable Care Act (ACA) and responding to funding reductions, regulatory changes, or weak organizational systems. An initial goal may be simple and broad, with further analysis and detailing as needed.

\section{Change scope}

Planned organizational change can be categorized based on three levels of complexity.

Developmental change involves adjustments to existing operations or improving a skill, method, or processessentially improving something that already exists. Examples include simple problem solving and routine training. Transitional change involves implementing something new and abandoning old ways of functioning. Examples might include implementing a Continuous Quality Improvement (CQI) system or a simple reorganization of staff roles. Transformational change requires "a radical shift of strategy, structure, systems, processes, or technology... so significant that it requires a shift of culture, behavior, and mind-set... to implement and sustain over time" (19).

COVID-19 challenges vary from developmental to transformational change. While adopting a new piece of PPE might be seen as simply developmental change, COVID-19 conditions clearly demand transformational change. This will be a nonlinear process, perhaps with occasional chaos; ideally including the use of a planned change process, with extensive involvement of many staff and comprehensive communication tools. A new future state for hospitals cannot be fully envisioned in advance. This paper reflects this dilemma: change in the COVID-19 era involves incomplete maps without clear destinations.

\section{Change strategies}

Examining organizational change in the COVID-19 era can also assess overall strategies used by change leaders. Empirical-rational strategies include change leaders sharing relevant data to show what needs to be done. Normative- 
reeducative strategies typically involve communications with employees to help them understand the needs for change and soliciting their support. Power-coercive strategies suggest using power, through directive leadership and "sanctions for noncompliance with the proposed change" (16). Current literature suggests that the first two, separately or in combination, are more appropriate than the third for successful change implementation.

\section{Change tactics}

Finally, evidence-based organization change tactics can be used as criteria to assess the extent to which organizational changes in response to COVID-19 fit with suggestions in the literature that their use should help improve prospects for success (20). The following change tactics can be used by change leaders.

(I) Clearly and persuasively communicate the need for and desirability of the change.

(II) Clearly and persuasively communicate the urgency for the change.

(III) Share information to document the change problem to be addressed.

(IV) Clearly communicate the vision and outcomes for the change.

(V) Clarify the plan for how the change process will be implemented

(VI) Provide top management support and commitment,

(VII) Select a cross section of employees for a team to guide the change.

(VIII) Provide for staff the information and training needed to implement the change.

(IX) Involve and solicit for their support key individuals and groups affected by the change.

(X) Ensure widespread participation of staff in the change process

(XI) Provide adequate opportunities for team building and conflict management.

(XII) Ensure support by political overseers or governing boards.

(XIII) Ensure support by collaborating organizations and other community partners.

(XIV) Clearly communicate progress on the change process throughout the organization

(XV) Provide sufficient resources (staff time, necessary funding) for the change process.

(XVI) Ensure that any concerns of staff are addressed.
(XVII) Use monitoring tools to track progress and results.

(XVIII) Revise project activities as appropriate based on new information or changing conditions.

(XIX) Institutionalize the results of the change process through formal changes in policies and procedures, training, new or modified staff roles, etc.

(XX) Evaluate the results of the change process using data

(XXI) Make staff aware of the results of the change process.

The relevance of the possible use of these tactics during the current crisis will be discussed in a later section.

All these elements_change leadership competencies, the scope of change needed, change strategy, readiness and capacity for change, and change tactics - can all be used as criteria for assessing the relevance of their use during the COVID-19 crisis.

\section{The complex/adaptive approach to change}

During the COVID-19 crisis, change leadership demands quick, complex decision making with little or incomplete information. Leaders need to employ change approaches and thinking that matches the complexity and ambiguity of the challenges currently facing them, their clinical teams, and their organization. In addition to the change leadership skills and change principles discussed above, the current crisis requires the new ways of thinking and leading proposed through the study and organizational applications of CAS (21), discussed next.

Critics of the planned approach to change argue that it relies heavily on information era assumptions about what we know and don't know $(22,23)$. Traditional models suggest that change generally proceeds in a logical way towards ends desired by the initiator of the change effort, but others claim that the highly interconnected and rapidly changing knowledge era can be better understood through applications of insights gained from complexity disciplines in science and mathematics (22). Complexity thinking has been applied to both organizational change and leadership in healthcare since the 1990's, and recent reviews of healthcare change/leadership literature highlight general acceptance of the characterization of healthcare as a CAS (24-26). However, it is also important to note that, like planned change, the complex/emergent approach has few empirical studies that speak to conditions that ensure effective outcomes. It is more descriptive than prescriptive 
and thus lacks clear guidance to those living through change. Challenges also include applying knowledge of the natural, biotic world to conscious, human systems (24). This summary of the complexity approach to change assumes application to human systems, replacing "agents" and "elements" with "actors" or "players".

Most change and leadership theorists using this approach refer to mechanisms underlying CAS found in the natural world $(27,28)$. Critical elements that comprise CASs include high levels of interconnected, interdependent action between many players (25). It has been said that computers are complicated, but mayonnaise is complex. Computers can be taken apart; individual components be examined independently. But mayonnaise is blended such that elements are no longer separate. In healthcare, the complex mix includes patients, providers at all levels, administrators, hospitals, medical groups, clinics, insurance companies and regulatory bodies all intertwined together in the delivery of care. The actions of one can have unanticipated consequences elsewhere. As a result, CASs include high levels of uncertainty and low levels of agreement about the source of systemic tensions and solutions for resolving them.

In ordered systems, managers can determine the desired end state, assess the initial situation and consequently set out a series of actions to reach the desired end state. In unordered systems, one cannot do so because of the uncertainty related to how the end state can be attained (24).

For instance, medical group administrators assumed that urgent care visits would dramatically increase during COVID-19. They predicted this would offset some early drop-offs reported in patient visits to primary care and they proactively trained additional staff . However, patients elected to avoid all but the most urgent healthcare settings, which was completely unprecedented. Paradoxically, healthcare systems are financially struggling as a result of lowered patient visits even as they face the greatest health crisis of our time.

Change in CAS occurs through adaptation (27). As the system becomes destabilized; it shifts in response to internal and external pressures. Sometimes, with enough threat, systems can shift quite quickly. Medical groups and clinics moved to telehealth within weeks of the arrival of COVID-19. Some organizations had been considering telehealth for years, and 10-month roll-out plans were underway. This kind of adaptation is rare, and reflects the intensity of the destabilizing impact of COVID-19. More often, iterative experimentation and learning support change that unfolds more slowly as new patterns emerge (24). A COVID-19 example includes first the temporary and then chronic shortages of PPE which led to shifts in regulatory policy on reuse, which further destabilized the system as long held personal safety practices were upended and the responsibility for safety shifted from regulatory oversight to individual practice. Finally, adaptation is enhanced when people through a variety of differing knowledge bases and perspectives act together to solve problems. Neighbors began sewing masks and giving them away to keep each other safe, protect healthcare workers, and save N95 masks for those who really needed them. Finally, CASs are organized around simple rules that govern behavior. Bedside nurses' top priority is keeping patients safe through the delivery of care, while administrators are responsible for the financial health and efficiency of the institution. While there is inherent tension between these two, prior to the pandemic, they generally lived in a negotiated equilibrium.

Perhaps the greatest challenge for human CAS is our own consciousness. Biotic systems shift over time; new elements come into being or fade away without the pain and suffering that human systems experience with disruption (29). We are just beginning to understand the toll on healthcare systems and workers, and the long term mental health challenges they may face.

Change in CAS suggests that managers can influence (versus control) adaptation through recognizing and attending to these mechanisms. One approach, Adaptive Leadership $(28,30)$ builds upon knowledge of CAS and creates a change leadership framework for highly complex environments and change situations. Several authors offer leadership-focused suggestions for influencing CAS related change.

* See the system: change begins with the change initiator's ability to take a broad view of the system they are in. What are the multiple, interacting dynamics? Without looking for blame, adaptive problems must be assessed with the largest possible systems perspective. "Getting on the balcony" involves stepping away from the action of the stage and viewing the challenge from an outsider perspective.

* Regulate distress: managers and change leaders have to pay attention to and regulate distress. This includes tolerating tension, conflict and stress so it stays productive and not overwhelming. Shapiro and Carr (29) suggested approaches to help people by recognizing their distress, providing 
empathetic support, and encouraging new ways of understanding their changing context.

* Bring people together: connect diverse perspectives together, provide minimal structure, and then empower others to be creative and solve problems $(28,31)$.

* Engage in iterative cycles of experimentation: improvise and experiment, continue to assess the impacts of what's tried, reflect and question to support emergence of novel processes, connections and solutions.

* Encourage/protect leadership at all levels: stay open to the ideas of others, and especially people who may be "at the fringe" or in low status positions.

Complexity leadership is a multi-level process that engages the entire system rather than focusing on attributes of an individual. Leadership roles shift from planning, organizing and engaging others in the effort to a focus on framing critical issues and questions, connecting people and processes in novel ways, articulating and incorporating real-time learning and attending to what's emerging as the pandemic unfolds.

Next, after a review of the COVID-19 crisis and the impacts to our healthcare system and institutions, a model for determining use of traditional and crisis/complexity driven approaches for continued navigation of the COVID-19 crisis in healthcare will be offered.

\section{The crisis of COVID-19: current change issues and challenges}

The COVID-19 pandemic brought a series of local crises to healthcare delivery organizations across the country and tested the system in a novel way. COVID-19 is a crisis in the truest sense: the needs of the system overwhelmed its resources and decisions were made based on a "lowest harm" rather than "highest quality" paradigm. In a shortterm crisis, such as a weather or mass casualty event, or even a localized infectious disease outbreak, the parameters of the crisis are obvious and staff are usually immediately aware of the switch from "normal operations" to a war-time mentality of harm reduction critical to crisis management. These crises are time- and resource-limited, often resolved within a few days and with unmet needs filled quickly by local resources.

Traditional crisis management approaches essentialize critical services and assume a return to normal at some point in the not-too-distant future. Such strategies focus on core functions designed to maintain safety during times of heavily unmet need, such as increasing bed availability, managing staffing shortages and changing competencies, and dealing with critical shortages of equipment and supplies (32). As a long-term, relapsing and remitting crisis, COVID-19 presents an altogether different set of challenges to the crisis management model as traditionally superimposed on an organizational change infrastructure. In a crisis management model in which a return to "normal" is anticipated in a period of days to weeks, holding off on "elective" surgeries or routine procedures might be seen as low-risk. COVID-19 brings a unique set of challenges as there is no anticipated, sustained return to normal in the near future, necessitating a new framework for the provision of care for the chronically and acutely ill that balances the benefits and harms of both routine organizational change and crisis management models (33). This pandemic created systemic uncertainty on all fronts: an unplanned crisis in both scope and duration. It is more than just a crisis of delivery; the complex and unknown nature of it necessitates a potentially foundational organizational change approach.

The need for proactive, robust crisis management protocols and a wider discussion about the ethical implications of a shift to a crisis management paradigm has been recognized for years. More important than adhering strictly to a single ethical framework or decision-making protocol, there is a need for transparency and a commitment to incorporating ethical principles into every step of the crisis response. As the organization shifts from routine daily operations to change management to contingency operations to crisis management, the ethical considerations will also shift and require ongoing communication and careful deliberation. Even prior to the COVID-19 pandemic, a strong movement from within healthcare leadership pushed for proactive crisis management strategies with strong ethical underpinnings. Consensus statements from prominent experts both recognized the lack of preparedness for ethical crisis management and implored others to begin a national discussion about the need for robust, efficient, and ethical frameworks to guide actions during crisis and provide legal and psychosocial support for care providers (34). This is a problem that exists outside of the U.S. healthcare system as well; nursing researchers in Australia recommended a national dialogue regarding standards of care during a crisis and the need for a proactive debate regarding the ethical implications of crisis situations (35). In the months since the pandemic, consensus guidelines have emerged to provide ethical and legal cover for decision-making in 
resource-scarce environments, such as governing the use of ventilators (36), though these consensus guidelines are not a substitute for nationally- or globally-coordinated decisionmaking support for crisis management.

Despite the calls for national action, a recent systematic review of the Crisis Standards of Care (CSC) documents in each U.S. state found all but five states to be deficient in meeting the recommendations from the 2009 Institute of Medicine report providing guidelines for establishing CSC plans (37). Of the guidelines reviewed, only two states (Colorado and Minnesota) provided documents that could be considered exemplars. These deficiencies are not without consequences when an actual crisis emerges. While not done in the U.S., a large study of hospitals in London reported that the lack of national cohesion in the U.K. around triage policy and decision support guidelines resulted in unnecessary limitations to routine access to medical care, and potentially led to preventable deaths at the height of the initial COVID-19 outbreak (38).

The success of crisis management in the healthcare system is contingent upon the leadership skills of those involved in decision-making at the local level. Researchers have found that inclusive leadership styles substantially reduce the burden of psychological distress during pandemics $(39,40)$. They suggest three primary distinctions in a successful crisis management paradigm: decreased individual autonomy, which provides moral cover and ethical clarity through set standards during the crisis; a laser focus on prognostic factors and maximizing benefit; and transparent communication up and down the chain of command. Health systems that have navigated the pandemic successfully have relied on transparent and honest decision making by accessible leaders, representing another "lesson learned" during COVID-19 that should be carried into the collective future (41).

A focus on ethical considerations and sensitivity to the ethical climate may have serious implications in the development of psychological distress among clinicians. One study found that the clinicians' perception of ethical decision-making during the COVID-19 pandemic was independently associated with anxiety, depression, and burnout (42). Even in the best of circumstances, the psychological effects of crisis decision-making on frontline clinicians cannot be overstated and was predicted by some. In one study of a mock implementation of CSC at an urban hospital in 2018, clinicians reported at the conclusion of the exercise feeling a sense of moral ambiguity and challenges to their roles and identities as clinicians when putting the crisis protocols into simulated practice (43).

There have been (and will continue to be) several competing ethical decisions at play during the COVID-19 pandemic, such as balancing the public health, chronic health, and acute care needs of the population. Within the oncology community, surgeons have been forced to weigh the relative benefit of early cancer diagnosis and treatment with the risks of a new Covid infection from "elective" procedures in the hospital. Cancer patients and their doctors have been left to decide whether a fourfold increase in the risk of death due to COVID-19 is preferable to a $20 \%$ increase in risk of cancer death, which, according to some, is like choosing between "the devil and the deep sea" (44). Rehabilitation physicians in Scotland, concerned about the latent effects of the pandemic, proposed a 5 -stage crisis management approach that utilizes a longer-term harm reduction model of signal detection, prevention/preparedness, damage containment, recovery, and learning (45). The strength of such a model is its assumption of "down time" between periods of acute illness/crisis; its limitation is its unclear relationship to normal organizational change modes of operation during the periods of relative calm.

A continuum model for crisis management (46) noted that critical incidents may require a "range of responses" for frontline providers, allowing for resource scarcity to drive specific actions and providing a framework for resource allocation based on ethical principles. Their crisis management model is based on a single mass casualty event and allows end-users to pivot between three levels of decision-making: conventional, in which resources and needs are relatively aligned; contingency, in which resources are misaligned but still allow for the provision of "functionally equivalent care;" and crisis, in which resources and needs are so misaligned that the provision of care is based on the best level possible, "given the resource gap." Central to the ability to pivot between these ethical and functional crisis management frameworks is the recognition of a triggering event for resource scarcity and transparency around decision-making frameworks which are not always apparent in traditional organizational change models. In another approach to equitable resource allocation during a period of scarcity, Knebel et al. (47) argued that a separate resource distribution process should exist for each scarce resource, with decision-making based on values held at the local and individual level. Others have recommended the deployment of specialized "resource allocation teams" who can make ethically-challenged clinical decisions in real time 
during periods of crisis causing resource scarcity (48).

There is a strong sense that many of the innovations enabled by the rapid decision-making processes inherent to the COVID-19 crisis ought to be sustained, such as the sharp turn towards telemedicine and reduction of several administrative barriers to patient care. Personal accounts of the success of rapid testing and implementation of new technologies, abandoned administrative red tape, and a strong focus on patient outcomes as the driving force for care are evident in the emerging literature (49). While COVID-19 has laid bare several flaws in the US healthcare system, the innovative strategies, teamwork, and crisis management models that have risen to the challenge may light the way for deeper systemic change in the long term.

\section{Planned, crisis driven, and adaptive change during Covid}

Given the multi-layered, on-going crisis that COVID-19 presents in hospitals and other healthcare institutions, an equally multi-layered and responsive change approach is necessary. In addition to national, systemic issues that include shortages of testing, PPE, and other resources, experiences thus far with COVID-19 have raised critical issues to solve at the local hospital level. Crisis management reveals pressing ethical challenges and competing ethical stances, rising anxiety and potential mental health challenges for providers, quickly changing guidelines as COVID-19 knowledge increases, all requiring local change management solutions. Such solutions rely on the leadership competencies of both formal and informal leaders. UhlBien et al. (22) suggest that leadership falls into three broad categories: traditional bureaucratic leadership; leadership that encourages creativity and learning through CAS; and an organization-wide dynamic that gives rise to emergent change, with the latter including adaptive leadership. In a similar vein, Belrhiti (24) suggests that formal leaders assess situations for use of traditional or complexity leadership. Issues that lend themselves to problem solving models can be addressed using transactional or transformational leadership approaches. Situations characterized by uncertainty require complexity thinking and adaptive approaches. Leadership and management demands during COVID-19 will continue to include fundamental, informed choice of change and leadership strategy based on rising and falling levels of ambiguity and crisis.

Issues that become clear during a crisis can be addressed using a combination of change approaches depending on the certainty of knowledge and clarity of implementation. The complex/adaptive approach utilizes insights and approaches found in the crisis and adaptive leadership literature. This is probably most useful during times of crisis which require constant assessment; ongoing high levels of communication; iterative cycles of experimentation, reflection and learning; and bringing multiple disciplines and stakeholders together and attention to/mitigation of distress. The planned approach to change is probably most useful for implementing new practices and processes that have become best practices. This requires a relatively high degree of certainty and agreement between leaders across disciplines to ensure success, and may be best accomplished during times of COVID-19 remission. Each suggests clear actions and roles that formal and informal leaders may engage in that support responsiveness in either situation. Differences between the two are summarized in Table 1 .

Planned change offers a guided map for senior and middle level managers as they consider implementation of emerging best practices in care delivery and crisis management during times of COVID-19 remission. Adaptive change gives managers clues for ways to respond during periods of recurrent crisis. Woven together, they provide a more comprehensive framework than either provides alone.

\section{Conclusions}

In the COVID-19 crisis environment, traditional models of organizational change clearly need reexamination in terms of their relevance, especially in healthcare. Typically, planned organizational change would be launched only after a full assessment of the problem and then assessment of internal readiness and change capacity factors, but the extreme time demands and crisis conditions of COVID-19 make full assessments and detailed advanced planning of the change process more difficult. Regardless, some of the organizational change tactics discussed earlier, such as describing the problem and the need for and urgency of change, are self-evident and will happen almost automatically. Stating clear outcomes of the change may not be as simple as it seems. Is the goal to return the hospital to the former "normal", or is the goal to achieve some "new normal?" Plans for implementing many of the tactics are ideally developed in some detail before launching the process, but this is less likely to occur in crisis conditions. Announcing a formal change process and outlining a formal action system including action teams or a problem-solving group processes might be rushed, with implementation 
Table 1 Planned change and crisis/adaptive change

\begin{tabular}{|c|c|}
\hline Planned change & Crisis change/adaptive/complexity \\
\hline $\begin{array}{l}\text { Assessment: change readiness and capacity; done once to support } \\
\text { change planning }\end{array}$ & $\begin{array}{l}\text { Evaluate the system: On-going "on the balcony": ongoing } \\
\text { assessment of how the org is responding }\end{array}$ \\
\hline Goals: tangibles about what we are striving to achieve & $\begin{array}{l}\text { Simple rules: clarify what is most important; Provide direction for } \\
\text { hard choices daily }\end{array}$ \\
\hline Strategy: empirical-rational; normative-reeducative & $\begin{array}{l}\text { Experimentation and communication: iterative cycles of action/ } \\
\text { reflection/discussion/planning }\end{array}$ \\
\hline
\end{tabular}

CAS, Complex Adaptive Systems.

happening with little advanced planning. Even in a crisis, spending time to set up these systems and clearly articulating the values driving decision-making can give staff clarity and perhaps reduce stress caused by uncertainty. The early inadequacies of PPE and ventilators vividly showed the importance of providing necessary resources; and urgent needs for cross training staff showed another limitation of the traditional approach. Changes to initial plans can be expected and should be clearly communicated. Data from monitoring tools can guide change leaders in making timely changes as needed. Informing staff of intermediate accomplishments or changes can give them a sense of progress and perhaps optimism. Evaluation of the effects of changes will also be rushed, but short feedback loops will enable additional adaptations or changes to be made quickly.

Even if the process is rushed, research suggests that participative management styles including structures for obtaining input are likely to enhance prospects for success, and can be quickly be established if the change leaders can effectively use participative leadership theories. As discussed above, Adaptive Leadership seems especially appropriate, as does transformational leadership (50), with its principles of idealized influence by trusted and admired leaders, inspirational motivation and visioning, intellectual stimulation to encourage innovation and question assumptions, and individualized consideration through coaching and supporting staff.
The COVID-19 crisis is forcing reexamination of other aspects of traditional organizational change. Organizational culture change, a popular change activity for decades, is typically a multi-year process. Culture changes in this crisis context are probably quick and not consciously considered, as staff are forced into new behaviors, interaction patterns, and norms as they quickly analyze and solve problems and institutionalize new ways of working, while recognizing the need for near constant change. If change leaders can give some thoughtful attention to how existing norms, roles, and values are being affected, they can initiate critical dialogue about how these changes can be more intentional in addressing quality standards and staff morale while working within time pressures and resource inadequacies.

Perhaps one of the greatest challenges of this moment is the realization that there may not be a post-COVID-19 state in the healthcare system. The organizational change and crisis management models of the past need to find a new articulation, where periods of relative calm and interspersed crisis are proactively recognized and managed. The health system needs both sustained, "big picture" changes driven by organizational change approaches, with the flexibility, readiness, and ethical considerations of crisis management strategies built in.

\section{Acknowledgments}

The authors acknowledge all the committed and courageous 
health care managers and staff on all levels living on the front line of complexity and crisis. We will all be learning from and making sense of their experiences and valiant efforts for years to come.

Funding: None.

\section{Footnote}

Provenance and Peer Review: This article was commissioned by the Guest Editors (Erick Guerrero and Jemima A. Frimpong) for the series "Organizational Approaches to Implement Rapid Change in Hospitals to Respond to Public Health Emergencies" published in Fournal of Hospital Management and Health Policy. The article has undergone external peer review.

Conflicts of Interest: All authors have completed the ICMJE uniform disclosure form (available at http://dx.doi. org/10.21037/jhmhp-21-11). The series "Organizational Approaches to Implement Rapid Change in Hospitals to Respond to Public Health Emergencies" was commissioned by the editorial office without any funding or sponsorship. The authors have no other conflicts of interest to declare.

Ethical Statement: The authors are accountable for all aspects of the work in ensuring that questions related to the accuracy or integrity of any part of the work are appropriately investigated and resolved.

Open Access Statement: This is an Open Access article distributed in accordance with the Creative Commons Attribution-NonCommercial-NoDerivs 4.0 International License (CC BY-NC-ND 4.0), which permits the noncommercial replication and distribution of the article with the strict proviso that no changes or edits are made and the original work is properly cited (including links to both the formal publication through the relevant DOI and the license). See: https://creativecommons.org/licenses/by-nc-nd/4.0/.

\section{References}

1. Hughes M. Leading changes: Why transformation explanations fail. Leadership 2016;12:449-69.

2. Scott A, Sivey P, Ait Ouakrim D, et al. The effect of financial incentives on the quality of health care provided by primary care physicians. Cochrane Database Syst Rev 2011;(9):CD008451.

3. Lee SY, Alexander J. Consequences of Organizational Change in U.S. Hospitals. Med Care Res Rev 1999;56:227-76.

4. Bazzoli GJ, Dynan L, Burns L, et al. Two Decades of Organizational Change in Health Care: What Have We Learned? Med Care Res Rev 2004;61:247-331.

5. Conrad DA, Grembowski D, Hernandez S, et al. Emerging lessons from regional and state innovation in value-based payment reform: balancing collaboration and disruptive innovation. Milbank Q 2014;92:568-623.

6. Herbst T, Foerster J, Emmert M. The impact of pay-forperformance on the quality of care in ophthalmology: Empirical evidence from Germany. Health Policy 2018;122:667-73.

7. Yee CA, Pizer S, Frakt A. Medicare's Bundled Payment Initiatives for Hospital-Initiated Episodes: Evidence and Evolution. Milbank Q 2020;98:908-74.

8. Karim SA, Nevola A, Morris M, et al. Financial Performance of Hospitals in the Appalachian Region Under the Hospital Readmissions Reduction Program and Hospital Value-Based Purchasing Program. J Rural Health 2021;37:296-307.

9. Cross DA, Cohen G, Harris Lemak C, et al. Sustained participation in a pay-for-value program: impact on highneed patients. Am J Manag Care 2017;23:e33-e40.

10. Boyal A, Hewison A. Exploring senior nurses' experiences of leading organizational change. Leadersh Health Serv (Bradf Engl) 2016;29:37-51.

11. Øygarden O, Olsen E, Mikkelsen A. Changing to improve? Organizational change and change-oriented leadership in hospitals. J Health Organ Manag 2020. doi: 10.1108/JHOM-09-2019-0280.

12. Bradley EH, Brewster AL, McNatt $Z$, et al. How guiding coalitions promote positive culture change in hospitals: a longitudinal mixed methods interventional study. BMJ Qual Saf 2018;27:218-25.

13. Jakobsen MD, Clausen T, Andersen LL. Can a participatory organizational intervention improve social capital and organizational readiness to change? Cluster randomized controlled trial at five Danish hospitals. J Adv Nurs 2020;76:2685-95.

14. Packard T. Organizational change: A conceptual framework to advance the evidence base. J Hum Behav Soc Environ 2013;23:75-90.

15. Holt DT, Armenakis AA, Feild HS, et al. Readiness for Organizational Change. J Appl Behav Sci 2007;43:232-55.

16. Burke W. Organization change: Theory and practice. 5 th ed. Thousand Oaks, CA: Sage Publications; 2018.

17. Lehman WEK, Greener JM, Simpson DD. Assessing organizational readiness for change. J Subst Abuse Treat 
2002;22:197-209.

18. Judge W, Douglas T. Organizational change capacity: the systematic development of a scale. Journal of Organizational Change Management 2009;22:635-49.

19. Anderson D, Anderson LA. Conscious change leadership: Achieving breakthrough results. Leader to Leader 2011;2011:51-9.

20. Packard T, Shih A. Organizational change tactics: The evidence base in the literature. J Evid Based Soc Work 2014;11:498-510.

21. Angeli F, Montefusco A. Sensemaking and learning during the COVID-19 pandemic: A complex adaptive systems perspective on policy decision-making. World Dev 2020;136:105106.

22. Uhl-Bien M, Marion R, Mckelvey B. Complexity Leadership Theory: Shifting leadership from the industrial age to the knowledge era. Leadersh Q 2007;18:298-318.

23. Stacey R. The challenge of human interdependence. European Business Review 2007;19:292-302.

24. Belrhiti Z, Nebot Giralt A, et al. Complex Leadership in Healthcare: A Scoping Review. Int J Health Policy Manag 2018;7:1073-84.

25. Kannampallil TG, Schauer GF, Cohen T, et al. Considering complexity in healthcare systems. J Biomed Inform 2011;44:943-7.

26. Khan S, Vendermorris A, Shepherd J, et al. Embracing uncertainty, managing complexity: applying complexity thinking principles to transformation efforts in healthcare systems. BMC Health Serv Res 2018;18:192.

27. Marion R. Complexity theory for organizations and organizational leadership. In: Uhl-Bien M, Marion $\mathrm{R}$, editors. Complexity Leadership part 1: conceptual foundations. Charlotte: Information Age Publishing; 2008.

28. Lichtenstein B, Plowman D. The leadership of emergence: A complex systems leadership theory of emergence at successive organizational levels. Leadership Quarterly 2009;20:617-30.

29. Shapiro E, Carr A. Lost in Familiar Places: Creating New Connections Between Individuals and Society. New Haven: Yale University Press; 1991.

30. Heifetz R, Linsky M, Grashow A. The practice of Adaptive Leadership. Boston: Harvard Business Press; 2009.

31. Schreiber C, Carley K. Dynamic network leadership: Leading and learning for adaptability. In: Uhl-Bien M, Marion R, editors. Complexity leadership Part 1: Conceptual foundations. Charlotte: Information Age Publishing, Inc.; 2008.

32. Duley MG. The Next Pandemic: Anticipating an
Overwhelmed Health Care System. Yale J Biol Med 2005;78:355-62.

33. Khazanie P, Wynia M, Dickert N. Forced ChoicesEthical Challenges in Cardiology During the COVID-19 Pandemic. Circulation 2020;142:194-6.

34. Christian MD, Sprung CL, King MA, et al. Triage: care of the critically ill and injured during pandemics and disasters: CHEST consensus statement. Chest 2014;146:e61S-74S.

35. Cusack L, Gebbie K. Call for national dialogue: Adapting standards of care in extreme events. We are not ready. Collegian 2017;24:93-100.

36. Joebges S, Biller-Andorno N. Ethics guidelines on COVID-19 triage-an emerging international consensus. Critical Care 2020;24:201.

37. Romney D, Fox H, Carlson S, et al. Allocation of Scarce Resources in a Pandemic: A Systematic Review of US State Crisis Standards of Care Documents. Disaster Med Public Health Prep 2020;14:677-83.

38. Burman R, Cairns R, Canestrini S, et al. Making ordinary decisions in extraordinary times. BMJ 2020;370:m3268.

39. Ahmed F, Zhao F, Faraz N. How and When Does Inclusive Leadership Curb Psychological Distress During a Crisis? Evidence From the COVID-19 Outbreak. Front Psychol 2020;11:1898.

40. Kirkpatrick JN, Hull S, Fedson S, et al. Scarce-Resource Allocation and Patient Triage During the COVID-19 Pandemic. JACC 2020;76:85-92.

41. Graham RN, Woodhead T. Leadership for continuous improvement in healthcare during the time of COVID-19. Clin Radiol 2021;76:67-72.

42. Azoulay E, De Waele J, Ferrer R, et al. Symptoms of burnout in intensive care unit specialists facing the COVID 19 outbreak. Ann Intensive Care 2020;10:110.

43. Chuang E, Cuartas P, Powell T, et al. "We're Not Ready, But I Don't Think You're Ever Ready.” Clinician Perspectives on Implementation of Crisis Standards of Care. AJOB Empir Bioeth 2020;11:148-59.

44. Chakraborty M, Pandey M. Caring for cancer patients in the Covid pandemic: choosing between the devil and deep sea. World J Surg Oncol 2020;18:220.

45. Khoo TC, Jesudason E, FitzGerald A. Catching our breath: reshaping rehabilitation services for COVID-19. Disabil Rehabil 2021;43:112-7.

46. Hick JL, Hanfling D, Cantrill S. Allocating Scarce Resources in Disasters: Emergency Department Principles. Ann Emerg Med 2012;59:177-87.

47. Knebel AR, Sharpe VA, Danis M, et al. Informing the Gestalt: An Ethical Framework for Allocating Scarce 
Federal Public Health and Medical Resources to States During Disasters. Disaster Med Public Health Prep 2014;8:79-88.

48. Kuschner WG, Pollard J, Ezeji-Okoye S. Ethical Triage and Scarce Resource Allocation During Public Health Emergencies: Tenets and Procedures. Hosp Top

doi: 10.21037/jhmhp-21-11

Cite this article as: Choflet A, Packard T, Stashower K. Rethinking organizational change in the COVID-19 era. J Hosp Manag Health Policy 2021;5:16.
2007;85:16-25.

49. Short JB, Mammen A. A Pandemic Application of Creative Destruction in Healthcare. Front Health Serv Manage 2020;37:4-9.

50. Bass B, Avolio B. Transformational leadership (2nd ed.). Mahwah, NJ: Lawrence Erlbaum Associates; 2006. 